\title{
Influence Of Library Environment And User Education On Undergraduates' Use Of Library At The University Of Ibadan, Nigeria
}

\author{
Oluyomı Folorunso \\ Esther Njoku \\ Department of Library, Archival and Information Studies, \\ University of Ibadan, Nigeria
}

doi: 10.19044/esj.2016.v12n19p288 URL:http://dx.doi.org/10.19044/esj.2016.v12n19p288

\begin{abstract}
The study investigated the influence of library environment and user education on undergraduates' use of the University central library at the University of Ibadan, Nigeria.The study adopted the survey research design and the population consisted of 18,034 regular undergraduates in ten faculties from which a sample size of 360 respondents was selected using the proportionate, random sampling technique. The research instrument used for data collection was a self-developed questionnaire while data was analysed using frequency distribution, percentages, mean and standard deviation together with regression and correlation analysis.The finding of the study revealed that library environment at the University of Ibadan was characterised by good library building (mean=3.75), seating arrangement (mean=3.59), availability of information resources (mean=3.54), and fair electricity supply while there was poor Internet connectivity and Wi-Fi within and outside the library (mean=2.39). Methods of delivering user education were library orientation for new students (64.3\%) and use of library course (53.4\%) with guided library tour. Most undergraduates used the library daily or weekly in order to study and read for examinations. However, inadequate technological facilities to use for electronic sources (mean=2.65), and poor network/internet connectivity to access electronic databases were some of the major challenges of using the University library. The study found a significant relationship between the independent variables (library environment and user education) and the dependent variable (use of library) ( $\mathrm{r}=.342, \mathrm{~N}=322, \mathrm{P}<.01)$; while both library environment and user education jointly and independently contributed to the use of library among the undergraduates. programmes given more priority in the University library in order to sensitise more students and draw their attention to the available resources and services while the environment of the University library (both
\end{abstract}


internal and external) should be made more conducive to always attract the users to the library.

Keywords: Library environment, User education, Library use, Undergraduates, University of Ibadan, Nigeria.

\section{Introduction}

The University library is playing a critical role in the expansion of higher education and research. It has become a centre for co-operation, promotion and service in education and research (Chowdhury, 2006). As a result, the libraries need to evolve from a rigid hierarchical structure to a process-oriented structure that promotes good partnership with the user community and also facilitates the design and implementation of quality information service that meets their needs.

The importance of library to undergraduate students in a University cannot be underestimated. For this reason, the Association of College and Research Libraries [ACRL] (2011) asserted that "access to adequate library services and resources is essential regardless of where students, faculty, and programs are located and that members of the distance learning community are entitled to library services and resources equivalent to those provided for students and faculty in traditional campus settings”. Unwin, Bolton and Stephens (1997) noted that without ready access to library service, students' learning experience may be unacceptably bound and controlled. Inappropriate accessibility of learning resources raises serious questions of offering "the same academic award to those students without equivalent access to learning resources”. Therefore, Ojedokun (2000) pointed out that the provision of library and information services to students (including the distant learners) is Central to quality education.

All libraries, particularly university libraries where there are people of intellect seeking information on daily basis to advance knowledge, adequate plans should be made to ensure that a strong and lasting relationship with their customers which comprise of researchers, faculty members and mostly undergraduate students. The essence of such relationship is to collaborate and partner with the users so as to design services that will meet their specific information needs, especially in this dynamic information age.

Undergraduate students are major stakeholders in the university environment. In fact, they constitute the largest percentage of the total population in a university. For instance, as at 2011, the population of undergraduate students (full-time) was 12,935 (University of Ibadan Pocket Statistics, 2011), which represented $51.52 \%$ of the total students/staff population. Therefore, the interest of this group of library users must be 
protected and well represented. The librarians need to move closer to these users more than ever before in order not to lose them to the virtual world of internet and Google. Establishing this form of collaboration will help the librarians to win the trust of these students and also repose confidence in them, while they also feel a great sense of belonging in the environment in which they are part.

Every organisation, irrespective of the nature and size, does not operate or exist in a vacuum but within a certain environment; and many of the forces that make up the environment usually create some challenges and opportunities which directly or indirectly affect the existence, operations, and survival or otherwise of the organization. Consequently, librarians and information professionals must be able to anticipate this changing library environment and develop the vision and competencies at all levels in their organisations/ institutions to embrace this dynamic future (Njanja, 2009).

The impact of environment on institutional performance and success cannot be underestimated, and as posited by Adeoye and Elegunde (2012), organisations are starting to recognise environmental performance as a source of competitive advantage. Just like other business organisations, academic libraries also operate within two major types of environment, that is, internal and external environment.

In the changing environment, the user needs a specific training in the use of library services, which is effected by the Information Technology. User education means, to educate the user or an introduction given to user to help them to make the best use of the library. When a user comes to library for the first time, he does not know about various activities of the particular library. In user education library staff introduces the user about their services, operations, rule-regulations etc.

User education has the potential to encourage and transform library and information users from dependent learners into independent, selfdirected, lifelong learners. Without information literacy which can be acquired through the user education, undergraduate students are condemned to lack of information, dependence upon others for access to knowledge and information, and even to acute levels of information anxiety (Adeniran, 2011).

The importance of library user education cannot be over emphasised, because there cannot be a connection between the users and the materials or tools without proper education been given to the user who may not have any prior knowledge or idea on the use of such materials or tools. It will be wise enough for the librarians to see every users of the library as a novice, and therefore provide a procedural way of educating or orientating them (Akinbola, 2007). 
User education is required in higher educational institutions because of the on-going proliferation of information resources and the variable methods of access. As such, most students are faced with diverse information choices in their academic endeavors. Information is available through many channels like libraries, special interest organizations, manufacturers and service providers, media and the internet. However, this information increasingly comes unfiltered, which raises questions about authenticity, validity, and reliability (Abubakar and Isyaku, 2012).

Effective user education programme helps to create a friendly atmosphere where the users and the librarian see themselves as partners in progress. User education is of immense benefits to both the user and the library. Ultimately, the benefits of user-librarian collaboration are numerous and engenders the ease of locating needed materials in the library by users; saving of the users time; expose the user to alternative sources of information on a particular subject of interest; ability of the user to make use of specialized reference materials like directories, encyclopaedias, abstracts and indexes (e.g. science citation index, dissertation abstracts international); sensitise and create awareness of the users to all their library rights and privileges; and acquisition of the life-long habit of self-discovery of information in the library and the world wide web (WWW).

In the information world, environmental changes are continuously putting new pressures on libraries' performance and relevance. To respond to these changes, libraries - particularly those within the educational and research institutions are now formulating and implementing strategies to reorganise and repackage the way information products and services are produced and disseminated to the users. For instance, some libraries are now changing the look and structure of the library buildings by repainting, and beautifying the library surroundings; while some other libraries for instance, Lagos State University Library are erecting new structures and equip them with modern facilities. Also, libraries are investing heavily in information technologies like computers, electronic information resources and wireless internet connectivity which compels the users to come around the library.

Emwanta and Nwalo (2013) noted that university libraries are charged with the provision of information services for users, acting in the changing academic environment. Therefore, the librarians need to liaise with library users, faculties, departments and lecturers to support effective teaching, learning, and research in the universities. These challenges require university libraries to offer user-friendly services and facilities.

\section{Research Questions}

The following are the research questions for the study: 
What are the characteristics of library environment in the University of Ibadan, Nigeria?

What are the methods of delivering user education in University of Ibadan?

What is the purpose of library use by undergraduates in University of Ibadan?

What is the frequency of library use by undergraduates in University of Ibadan?

What are the challenges of using the university library by the undergraduates?

\section{Hypotheses}

The following null hypotheses were tested in the study at 0.05 level of significance.

Ho1: There is no significant relationship between the library environment and use of library by undergraduates at the University of Ibadan.

Ho2: There is no significant relationship between user education and use of library by undergraduates at the University of Ibadan.

Ho3: There is no joint effect of library environment and user education on the use of library by undergraduates at the University of Ibadan.

Ho4: There is no relative effect of library environment and user education on the use of library by undergraduates at the University of Ibadan.

\section{Method}

The survey research design was used for this study because it is mostly used in applied social research. The survey method relies on a questionnaire instrument and is the most common method used in library and information science research (Aina, 2002).

The proportionate, random sampling technique was adopted for the study, which comprised 18,034 undergraduate students of University of Ibadan. A sampling fraction of $2 \%$ was used to derive a sample size for each faculty and this gave a total of 360 respondents. This sample size is justified by Krejcie and Morgan 1970 who recommended a sample size of 384 for a sample of 20,000 .

\section{Results}

Research question 1: What are the characteristics of library environment in the University of Ibadan, Nigeria?

Data in Table 1 represent the nature of library environment in the University of Ibadan. 
Table 1: Characteristics of library environment

\begin{tabular}{|c|c|c|c|c|c|c|c|c|}
\hline $\mathbf{S} / \mathbf{N}$ & Library environment & Excellent & $\begin{array}{l}\text { Very } \\
\text { good }\end{array}$ & Good & Fair & Poor & Mean & S.D \\
\hline 1. & Library building & $\begin{array}{l}85 \\
26.4 \% \\
\end{array}$ & $\begin{array}{l}116 \\
36.0 \% \\
\end{array}$ & $\begin{array}{l}84 \\
26.1 \% \\
\end{array}$ & $\begin{array}{l}31 \\
9.6 \% \\
\end{array}$ & $\begin{array}{l}6 \\
1.9 \% \\
\end{array}$ & 3.75 & 1.01 \\
\hline 2. & Seating arrangement & $\begin{array}{l}74 \\
23.0 \%\end{array}$ & $\begin{array}{l}88 \\
27.3 \%\end{array}$ & $\begin{array}{l}119 \\
37.0 \%\end{array}$ & $\begin{array}{l}37 \\
11.5 \%\end{array}$ & $\begin{array}{l}4 \\
1.2 \%\end{array}$ & 3.59 & 1.00 \\
\hline 3. & $\begin{array}{l}\text { Availability of library } \\
\text { information resources }\end{array}$ & $\begin{array}{l}60 \\
18.6 \%\end{array}$ & $\begin{array}{l}114 \\
35.4 \%\end{array}$ & $\begin{array}{l}95 \\
29.5 \%\end{array}$ & $\begin{array}{l}45 \\
14.0 \%\end{array}$ & $\begin{array}{l}8 \\
2.5 \%\end{array}$ & 3.54 & 1.03 \\
\hline 4. & Library furniture & $\begin{array}{l}59 \\
18.3 \%\end{array}$ & $\begin{array}{l}97 \\
30.1 \%\end{array}$ & $\begin{array}{l}108 \\
33.5 \%\end{array}$ & $\begin{array}{l}49 \\
15.2 \%\end{array}$ & $\begin{array}{l}9 \\
2.8 \%\end{array}$ & 3.46 & 1.04 \\
\hline 5. & Library personnel & $\begin{array}{l}37 \\
11.5 \%\end{array}$ & $\begin{array}{l}107 \\
33.2 \%\end{array}$ & $\begin{array}{l}128 \\
39.8 \%\end{array}$ & $\begin{array}{l}29 \\
9.0 \%\end{array}$ & $\begin{array}{l}21 \\
6.5 \%\end{array}$ & 3.34 & 1.01 \\
\hline 6. & $\begin{array}{l}\text { Temperature within the } \\
\text { library }\end{array}$ & $\begin{array}{l}58 \\
18.0 \% \\
\end{array}$ & $\begin{array}{l}77 \\
23.9 \% \\
\end{array}$ & $\begin{array}{l}88 \\
27.3 \% \\
\end{array}$ & $\begin{array}{l}75 \\
23.3 \% \\
\end{array}$ & $\begin{array}{l}24 \\
7.5 \% \\
\end{array}$ & 3.22 & 1.20 \\
\hline 7. & Lighting & $\begin{array}{l}47 \\
14.6 \% \\
\end{array}$ & $\begin{array}{l}65 \\
20.2 \%\end{array}$ & $\begin{array}{l}105 \\
32.6 \% \\
\end{array}$ & $\begin{array}{l}75 \\
23.3 \% \\
\end{array}$ & $\begin{array}{l}30 \\
9.3 \%\end{array}$ & 3.07 & 1.18 \\
\hline 8. & $\begin{array}{l}\text { Décor of the library } \\
\text { (Interior Designs) }\end{array}$ & $\begin{array}{l}36 \\
11.2 \% \\
\end{array}$ & $\begin{array}{l}58 \\
18.0 \% \\
\end{array}$ & $\begin{array}{l}121 \\
37.6 \% \\
\end{array}$ & $\begin{array}{l}85 \\
26.4 \% \\
\end{array}$ & $\begin{array}{l}22 \\
6.8 \% \\
\end{array}$ & 3.00 & 1.08 \\
\hline 9. & $\begin{array}{l}\text { Signage within and } \\
\text { outside the library }\end{array}$ & $\begin{array}{l}27 \\
8.4 \%\end{array}$ & $\begin{array}{l}73 \\
22.7 \%\end{array}$ & $\begin{array}{l}123 \\
38.2 \%\end{array}$ & $\begin{array}{l}71 \\
22.0 \%\end{array}$ & $\begin{array}{l}28 \\
8.7 \%\end{array}$ & 3.00 & 1.06 \\
\hline 10 & $\begin{array}{l}\text { Noise level within the } \\
\text { library }\end{array}$ & $\begin{array}{l}33 \\
10.2 \%\end{array}$ & $\begin{array}{l}73 \\
22.7 \%\end{array}$ & $\begin{array}{l}97 \\
30.1 \%\end{array}$ & $\begin{array}{l}89 \\
27.6 \%\end{array}$ & $\begin{array}{l}30 \\
9.3 \%\end{array}$ & 2.97 & 1.14 \\
\hline 11 & Electricity supply & $\begin{array}{l}29 \\
9.0 \% \\
\end{array}$ & $\begin{array}{l}57 \\
17.7 \% \\
\end{array}$ & $\begin{array}{l}90 \\
28.0 \% \\
\end{array}$ & $\begin{array}{l}85 \\
26.4 \% \\
\end{array}$ & $\begin{array}{l}61 \\
18.9 \% \\
\end{array}$ & 2.71 & 1.22 \\
\hline 12 & $\begin{array}{l}\text { Internet connectivity } \\
\text { within the library }\end{array}$ & $\begin{array}{l}18 \\
5.6 \%\end{array}$ & $\begin{array}{l}53 \\
16.5 \%\end{array}$ & $\begin{array}{l}77 \\
23.9 \%\end{array}$ & $\begin{array}{l}73 \\
22.7 \%\end{array}$ & $\begin{array}{l}101 \\
31.4 \% \\
\end{array}$ & 2.42 & 1.24 \\
\hline 13 & $\begin{array}{l}\text { Wi-Fi within and outside } \\
\text { the library }\end{array}$ & $\begin{array}{l}16 \\
5.0 \%\end{array}$ & $\begin{array}{l}43 \\
13.4 \%\end{array}$ & $\begin{array}{l}86 \\
26.7 \%\end{array}$ & $\begin{array}{l}83 \\
25.8 \%\end{array}$ & $\begin{array}{l}94 \\
29.2 \%\end{array}$ & 2.39 & 1.18 \\
\hline
\end{tabular}

It was revealed that among the characteristics of the library environment, the library building was rated highest (mean=3.75). More than $60 \%$ considered the building as either excellent or very good. The seating arrangement was rated next (mean=3.59) with about half of the respondents believing it was excellent or very good as well. Availability of library information resources (mean=3.54) and library furniture (mean=3.46) both received ratings that were close to the seating arrangement.

The least rated characteristics of the environment were electricity supply, Internet connectivity and Wi-Fi connections. Overall, there are remarkable differences in the ratings among the respondents. All characteristics received "excellent" ratings, ranging from 5\% for Wi-Fi connections, to more than $25 \%$ for the library building. Conversely, all characteristics also received poor ratings.

\section{Research question 2: What are the methods of delivering user education in University of Ibadan?}

Table 2 showed the methods of delivering user education to undergraduate in the main library of the University 
Table 2: Methods of delivering user education

\begin{tabular}{|l|l|l|l|l|}
\hline S/N & Methods of user education & Yes & No & Ranking \\
\hline 1 & Lectures & $87(27.0 \%)$ & $235(73.0 \%)$ & 4 \\
\hline 2 & Library Orientation for new students & $207(64.3 \%)$ & $115(35.7 \%)$ & 1 \\
\hline 3 & $\begin{array}{l}\text { Guided library tour (being taken round the } \\
\text { library to show the available resources and } \\
\text { service }\end{array}$ & $130(40.4 \%)$ & $192(59.6 \%)$ & 3 \\
\hline 4 & $\begin{array}{l}\text { Use of library course (GES 101: Use of } \\
\text { English) }\end{array}$ & $172(53.4 \%)$ & $150(46.6 \%)$ & 2 \\
\hline 5 & Basic bibliographic instruction & $54(16.8 \%)$ & $268(83.2 \%)$ & 6 \\
\hline 6 & Advanced bibliographic instruction & $33(10.2 \%)$ & $289(89.8 \%)$ & 7 \\
\hline 7 & One-on-one library use instruction & $75(23.3 \%)$ & $247(76.7 \%)$ & 5 \\
\hline
\end{tabular}

Library orientation (64.3\%) was the most evident method. More than half of the students received user education programmes through the Use of library course. Each of basic bibliographic, advanced bibliographic and oneon-one library use instructions was a method used to deliver user education to less than one-quarter of the students. Guided library tour (40.4\%) was fairly popular among the students.

\section{Research question 3: What is the purpose of library use by undergraduates in University of Ibadan?}

The purpose of library use by undergraduates at the University of Ibadan is shown in Table 3

The most prominent purpose of using the library was to study and read for examinations (mean=3.51). Many of the students also use the library to look for materials for assignments $($ mean $=3.19)$ and to do research work (mean=3.16).

Table 3: Purpose of library use by undergraduates

\begin{tabular}{|c|c|c|c|c|c|c|c|}
\hline $\mathbf{S} / \mathbf{N}$ & Purpose & SA & A & D & SD & Mean & S.D \\
\hline 1 & $\begin{array}{lccc}\begin{array}{l}\text { To study } \\
\text { examinations }\end{array} & \text { and } & \text { read } & \text { for } \\
\end{array}$ & $\begin{array}{l}195 \\
60.6 \%\end{array}$ & $\begin{array}{l}106 \\
32.9 \%\end{array}$ & $\begin{array}{ll}12 \\
3.7 \%\end{array}$ & $\begin{array}{l}9 \\
2.8 \%\end{array}$ & 3.51 & 0.70 \\
\hline 2 & Find materials for assignments & $\begin{array}{l}131 \\
40.7 \%\end{array}$ & $\begin{array}{l}136 \\
42.2 \%\end{array}$ & $\begin{array}{l}39 \\
12.1 \%\end{array}$ & $\begin{array}{l}16 \\
5.0 \%\end{array}$ & 3.19 & 0.83 \\
\hline 3 & $\begin{array}{l}\text { To do serious research work, e.g. } \\
\text { project, term paper }\end{array}$ & $\begin{array}{l}128 \\
39.8 \%\end{array}$ & $\begin{array}{ll}137 \\
42.5 \%\end{array}$ & $\begin{array}{ll}38 \\
11.8 \%\end{array}$ & $\begin{array}{l}19 \\
5.9 \%\end{array}$ & 3.16 & 0.85 \\
\hline 4 & $\begin{array}{l}\text { To consult reference materials } \\
\text { like encyclopedia, dictionaries }\end{array}$ & $\begin{array}{ll}111 \\
34.5 \%\end{array}$ & $\begin{array}{ll}128 \\
39.8 \%\end{array}$ & $\begin{array}{ll}51 \\
15.8 \%\end{array}$ & $\begin{array}{l}32 \\
9.9 \%\end{array}$ & 2.99 & 0.95 \\
\hline 5 & $\begin{array}{l}\text { To borrow materials for academic } \\
\text { work }\end{array}$ & $\begin{array}{l}109 \\
33.9 \%\end{array}$ & $\begin{array}{l}127 \\
39.4 \%\end{array}$ & $\begin{array}{l}46 \\
14.3 \%\end{array}$ & $\begin{array}{l}40 \\
12.4 \%\end{array}$ & 2.95 & 0.99 \\
\hline 6 & Consult librarians for information & $\begin{array}{ll}54 \\
16.8 \%\end{array}$ & $\begin{array}{ll}142 \\
44.1 \%\end{array}$ & $\begin{array}{l}93 \\
28.9 \% \\
\end{array}$ & $\begin{array}{ll}33 \\
10.2 \% \\
\end{array}$ & 2.67 & 0.87 \\
\hline 7 & $\begin{array}{l}\text { Make use of the wireless internet } \\
\text { connectivity (Wi-Fi) on my } \\
\text { device }\end{array}$ & $\begin{array}{l}50 \\
15.5 \%\end{array}$ & $\begin{array}{l}123 \\
38.2 \%\end{array}$ & $\begin{array}{l}86 \\
26.7 \%\end{array}$ & $\begin{array}{l}63 \\
19.6 \%\end{array}$ & 2.50 & 0.98 \\
\hline 8 & $\begin{array}{l}\text { Access electronic information } \\
\text { resources }\end{array}$ & $\begin{array}{l}45 \\
14.0 \%\end{array}$ & $\begin{array}{l}123 \\
38.2 \%\end{array}$ & $\begin{array}{l}89 \\
27.6 \%\end{array}$ & $\begin{array}{l}65 \\
20.2 \%\end{array}$ & 2.46 & 0.97 \\
\hline 9 & $\begin{array}{l}\text { Make use of computers and } \\
\text { internet access }\end{array}$ & $\begin{array}{l}45 \\
14.0 \%\end{array}$ & $\begin{array}{l}116 \\
36.0 \%\end{array}$ & $\begin{array}{l}95 \\
29.5 \%\end{array}$ & $\begin{array}{l}66 \\
20.5 \%\end{array}$ & 2.43 & 0.97 \\
\hline 10 & To consult newspapers & 34 & 129 & 92 & 67 & 2.40 & 0.93 \\
\hline
\end{tabular}




\begin{tabular}{|l|l|l|l|l|l|l|l|}
\hline & & $10.6 \%$ & $40.1 \%$ & $28.6 \%$ & $20.8 \%$ & & \\
\hline 11 & Group discussion with my course & 24 & 84 & 126 & 88 & 2.14 & 0.90 \\
& mates & $7.5 \%$ & $26.1 \%$ & $39.1 \%$ & $27.3 \%$ & & \\
\hline 12 & For relaxation or sleep & 23 & 37 & 97 & 165 & 1.75 & 0.92 \\
& & $7.1 \%$ & $11.5 \%$ & $30.1 \%$ & $51.2 \%$ & & \\
\hline
\end{tabular}

The purpose of relaxation or sleeping in the library is the least popular among the students as more than $80 \%$ either disagreed or strongly disagreed that they do so.

\section{Research question 4: What is the frequency of library use by undergraduates in University of Ibadan?}

Table 4 showed the frequency of library use by undergraduates in the University of Ibadan.

The frequency of library use to study and read for examinations far outweighs every other purpose of use among the undergraduates. About $60 \%$ use the library at least twice in a week just for this purpose. In comparison, less than $18 \%$ go there at least twice weekly as well to read newspapers or participate in a group discussion. Specifically, more than $50 \%$ of the students have never been to the library to read newspapers or participate in a group discussion.

Table 4: Frequency of library use by undergraduates

\begin{tabular}{|c|c|c|c|c|c|c|c|c|c|}
\hline $\mathbf{s} / \mathbf{n}$ & Frequency & Daily & $\begin{array}{l}\text { Twice a } \\
\text { week }\end{array}$ & Weekly & Monthly & Quarterly & Never & Mean & S.D \\
\hline 1. & $\begin{array}{l}\text { To study and read for } \\
\text { examination }\end{array}$ & $\begin{array}{l}104 \\
32.3 \% \\
\end{array}$ & $\begin{array}{l}88 \\
27.3 \% \\
\end{array}$ & $\begin{array}{l}56 \\
17.4 \% \\
\end{array}$ & $\begin{array}{l}15 \\
4.7 \% \\
\end{array}$ & $\begin{array}{l}26 \\
8.1 \% \\
\end{array}$ & $\begin{array}{l}33 \\
10.2 \% \\
\end{array}$ & 4.40 & 1.64 \\
\hline 2. & $\begin{array}{l}\text { Find materials for } \\
\text { assignments }\end{array}$ & $\begin{array}{l}59 \\
18.3 \%\end{array}$ & $\begin{array}{l}68 \\
21.1 \%\end{array}$ & $\begin{array}{l}57 \\
17.7 \% \\
\end{array}$ & $\begin{array}{l}45 \\
14.0 \%\end{array}$ & $\begin{array}{l}30 \\
9.3 \%\end{array}$ & $\begin{array}{l}63 \\
19.6 \%\end{array}$ & 3.66 & 1.76 \\
\hline 3. & $\begin{array}{l}\text { To do research work, } \\
\text { e.g. project, term paper }\end{array}$ & $\begin{array}{l}41 \\
12.7 \%\end{array}$ & $\begin{array}{l}76 \\
23.6 \%\end{array}$ & $\begin{array}{l}44 \\
13.7 \%\end{array}$ & $\begin{array}{l}28 \\
8.7 \%\end{array}$ & $\begin{array}{l}53 \\
16.5 \%\end{array}$ & $\begin{array}{l}80 \\
24.8 \%\end{array}$ & 3.33 & 1.81 \\
\hline 4. & $\begin{array}{lr}\text { To consult reference } \\
\text { materials } & \text { like } \\
\text { encyclopedia, } & \\
\text { dictionaries } & \end{array}$ & $\begin{array}{l}39 \\
12.1 \%\end{array}$ & $\begin{array}{l}72 \\
22.4 \%\end{array}$ & $\begin{array}{l}51 \\
15.8 \%\end{array}$ & $\begin{array}{l}23 \\
7.1 \%\end{array}$ & $\begin{array}{l}36 \\
11.2 \%\end{array}$ & $\begin{array}{l}101 \\
31.4 \%\end{array}$ & 3.23 & 1.86 \\
\hline 5. & $\begin{array}{l}\text { To borrow materials } \\
\text { for academic work }\end{array}$ & $\begin{array}{l}24 \\
7.5 \%\end{array}$ & $\begin{array}{l}61 \\
18.9 \%\end{array}$ & $\begin{array}{l}52 \\
16.1 \%\end{array}$ & $\begin{array}{l}36 \\
11.2 \%\end{array}$ & $\begin{array}{l}36 \\
11.2 \%\end{array}$ & $\begin{array}{l}113 \\
35.1 \%\end{array}$ & 2.95 & 1.76 \\
\hline 6. & $\begin{array}{ll}\begin{array}{l}\text { Make use of the } \\
\text { wireless }\end{array} & \begin{array}{l}\text { internet } \\
\text { connectivity } \\
\text { on my device }\end{array} \\
\end{array}$ & $\begin{array}{l}40 \\
12.4 \%\end{array}$ & $\begin{array}{l}47 \\
14.6 \%\end{array}$ & $\begin{array}{l}33 \\
10.2 \%\end{array}$ & $\begin{array}{l}23 \\
7.1 \%\end{array}$ & $\begin{array}{l}43 \\
13.4 \%\end{array}$ & $\begin{array}{l}136 \\
42.2 \%\end{array}$ & 2.79 & 1.90 \\
\hline 7. & $\begin{array}{l}\text { Make use of computers } \\
\text { and internet access }\end{array}$ & $\begin{array}{l}24 \\
7.5 \%\end{array}$ & $\begin{array}{l}53 \\
16.5 \%\end{array}$ & $\begin{array}{l}37 \\
11.5 \%\end{array}$ & $\begin{array}{l}33 \\
10.2 \%\end{array}$ & $\begin{array}{l}28 \\
8.7 \%\end{array}$ & $\begin{array}{l}147 \\
45.7 \%\end{array}$ & 2.67 & 1.80 \\
\hline 8. & $\begin{array}{l}\text { Consult librarians for } \\
\text { information }\end{array}$ & $\begin{array}{l}27 \\
8.4 \%\end{array}$ & $\begin{array}{l}34 \\
10.6 \%\end{array}$ & $\begin{array}{l}45 \\
14.0 \%\end{array}$ & $\begin{array}{l}29 \\
9.0 \%\end{array}$ & $\begin{array}{l}53 \\
16.5 \% \\
\end{array}$ & $\begin{array}{l}134 \\
41.6 \%\end{array}$ & 2.61 & 1.73 \\
\hline 9. & $\begin{array}{l}\text { Access electronic } \\
\text { information resources }\end{array}$ & $\begin{array}{l}25 \\
7.8 \%\end{array}$ & $\begin{array}{l}43 \\
13.4 \%\end{array}$ & $\begin{array}{l}34 \\
10.6 \%\end{array}$ & $\begin{array}{l}43 \\
13.4 \%\end{array}$ & $\begin{array}{l}32 \\
9.9 \%\end{array}$ & $\begin{array}{l}145 \\
45.0 \%\end{array}$ & 2.61 & 1.76 \\
\hline 10. & $\begin{array}{l}\text { Group discussion with } \\
\text { my course mates }\end{array}$ & $\begin{array}{l}24 \\
7.5 \%\end{array}$ & $\begin{array}{l}32 \\
9.9 \%\end{array}$ & $\begin{array}{l}36 \\
11.2 \%\end{array}$ & $\begin{array}{l}28 \\
8.7 \%\end{array}$ & $\begin{array}{l}23 \\
7.1 \%\end{array}$ & $\begin{array}{l}179 \\
55.6 \%\end{array}$ & 2.35 & 1.75 \\
\hline 11. & To consult newspapers & $\begin{array}{l}19 \\
5.9 \% \\
\end{array}$ & $\begin{array}{l}35 \\
10.9 \%\end{array}$ & $\begin{array}{l}34 \\
10.6 \% \\
\end{array}$ & $\begin{array}{l}26 \\
8.1 \% \\
\end{array}$ & $\begin{array}{l}33 \\
10.2 \% \\
\end{array}$ & $\begin{array}{l}175 \\
54.3 \% \\
\end{array}$ & 2.31 & 1.70 \\
\hline
\end{tabular}


Daily use of the library to study and read for examinations was more than $30 \%$ while the purposes of finding materials for assignments, doing research work, and consulting references ranged between 12 to 20 percent in each case. Every other purpose recorded less than 10 percent of daily use.

\section{Research question 5: What are the challenges of using the University library by the undergraduates?}

Table 5 showed the challenges to library use by undergraduates in the University of Ibadan.

Table5: Challenges to library use by undergraduates

\begin{tabular}{|c|c|c|c|c|c|c|c|}
\hline $\mathbf{s} / \mathbf{n}$ & Challenges & SA & A & D & SD & Mean & S.D \\
\hline 1 & $\begin{array}{l}\text { Inadequate technological facilities to } \\
\text { use for electronic sources }\end{array}$ & $\begin{array}{l}69 \\
21.4 \%\end{array}$ & $\begin{array}{l}116 \\
36.0 \%\end{array}$ & $\begin{array}{l}91 \\
28.3 \%\end{array}$ & $\begin{array}{l}46 \\
14.3 \%\end{array}$ & 2.65 & 0.97 \\
\hline 2 & $\begin{array}{l}\text { Poor network/internet connectivity to } \\
\text { access electronic databases }\end{array}$ & $\begin{array}{l}67 \\
20.8 \%\end{array}$ & $\begin{array}{l}120 \\
37.3 \%\end{array}$ & $\begin{array}{l}88 \\
27.3 \%\end{array}$ & $\begin{array}{l}47 \\
14.6 \%\end{array}$ & 2.64 & 0.97 \\
\hline 3 & $\begin{array}{l}\text { Most of the library collections are } \\
\text { outdated and not current }\end{array}$ & $\begin{array}{l}75 \\
23.3 \% \\
\end{array}$ & $\begin{array}{ll}110 \\
34.2 \% \\
\end{array}$ & $\begin{array}{l}83 \\
25.8 \%\end{array}$ & $\begin{array}{ll}54 \\
16.8 \% \\
\end{array}$ & 2.64 & 1.02 \\
\hline 4 & $\begin{array}{l}\text { Difficulty in locating materials from } \\
\text { the shelves }\end{array}$ & $\begin{array}{l}70 \\
21.7 \%\end{array}$ & $\begin{array}{l}98 \\
30.4 \%\end{array}$ & $\begin{array}{l}115 \\
35.7 \%\end{array}$ & $\begin{array}{l}39 \\
12.1 \%\end{array}$ & 2.62 & 0.96 \\
\hline 5 & $\begin{array}{l}\text { Poor lighting due to epileptic power } \\
\text { supply }\end{array}$ & $\begin{array}{l}72 \\
22.4 \%\end{array}$ & $\begin{array}{l}104 \\
32.3 \%\end{array}$ & $\begin{array}{l}90 \\
28.0 \%\end{array}$ & $\begin{array}{l}56 \\
17.4 \%\end{array}$ & 2.60 & 1.02 \\
\hline 6 & $\begin{array}{l}\text { Low awareness of the information } \\
\text { sources and services }\end{array}$ & $\begin{array}{l}46 \\
14.3 \%\end{array}$ & $\begin{array}{l}115 \\
35.7 \%\end{array}$ & $\begin{array}{l}114 \\
35.4 \%\end{array}$ & $\begin{array}{l}47 \\
14.6 \%\end{array}$ & 2.50 & 0.91 \\
\hline 7 & $\begin{array}{l}\text { Inadequate information resources in } \\
\text { the library }\end{array}$ & $\begin{array}{l}49 \\
15.2 \%\end{array}$ & $\begin{array}{l}104 \\
32.3 \% \\
\end{array}$ & $\begin{array}{l}116 \\
36.0 \%\end{array}$ & $\begin{array}{ll}53 \\
16.5 \%\end{array}$ & 2.46 & 0.94 \\
\hline 8 & $\begin{array}{l}\text { Inadequate reading space in the } \\
\text { library }\end{array}$ & $\begin{array}{l}51 \\
15.8 \%\end{array}$ & $\begin{array}{ll}77 \\
23.9 \% \\
\end{array}$ & $\begin{array}{l}122 \\
37.9 \%\end{array}$ & $\begin{array}{l}72 \\
22.4 \% \\
\end{array}$ & 2.33 & 0.99 \\
\hline 9 & $\begin{array}{l}\text { Poor customer relations among } \\
\text { library staff }\end{array}$ & $\begin{array}{l}33 \\
10.2 \%\end{array}$ & $\begin{array}{l}76 \\
23.6 \% \\
\end{array}$ & $\begin{array}{l}139 \\
43.2 \% \\
\end{array}$ & $\begin{array}{l}74 \\
23.0 \% \\
\end{array}$ & 2.21 & 0.91 \\
\hline 10 & $\begin{array}{l}\text { Inability to evaluate the usefulness of } \\
\text { information gathered }\end{array}$ & $\begin{array}{l}19 \\
5.9 \% \\
\end{array}$ & $\begin{array}{l}98 \\
30.4 \% \\
\end{array}$ & $\begin{array}{l}134 \\
41.6 \% \\
\end{array}$ & $\begin{array}{l}71 \\
22.0 \% \\
\end{array}$ & 2.20 & 0.85 \\
\hline 11 & Poor attitude of library staff to users & $\begin{array}{l}25 \\
7.8 \%\end{array}$ & $\begin{array}{l}69 \\
21.4 \% \\
\end{array}$ & $\begin{array}{l}146 \\
45.3 \%\end{array}$ & $\begin{array}{l}82 \\
25.5 \%\end{array}$ & 2.11 & 0.88 \\
\hline 12 & $\begin{array}{l}\text { Lack of professionalism among the } \\
\text { library staff }\end{array}$ & $\begin{array}{l}20 \\
6.2 \%\end{array}$ & $\begin{array}{l}71 \\
22.0 \%\end{array}$ & $\begin{array}{l}154 \\
47.8 \%\end{array}$ & $\begin{array}{l}77 \\
23.9 \%\end{array}$ & 2.11 & 0.84 \\
\hline
\end{tabular}

The students' responses did not discriminate widely across the numerous challenges encountered in using the library. In spite of this, inadequate technological facilities (mean=2.65), poor network/internet connectivity (mean=2.64) and outdated library collections (mean=2.64) were about the greatest challenges encountered while poor attitude of library staff and their lack of professionalism were jointly considered the least of all (mean=2.11).

More than half of all students agreed or strongly agreed that inadequate technological facilities, poor network/internet connectivity, outdated library collections, difficulty in locating library materials and poor lighting conditions constitute challenges to their use of the library. All other 
challenges were similarly agreed or strongly agreed upon by one-third to half of the respondents.

\section{Test of Hypotheses}

Ho 1: There is no significant relationship between the library environment and use of library by undergraduates at the University of Ibadan

Table 6 presents a correlation analysis showing the relationship between the library environment and use of library by undergraduates in the University.

Table 6: Relationship between the library environment and use of library

\begin{tabular}{|l|l|l|l|l|l|l|}
\hline Variable & Mean & Std. Dev. & $\mathbf{N}$ & $\mathbf{R}$ & $\mathbf{P}$ & Remark \\
\hline Use of library & 32.9068 & 13.2833 & & & & \multirow{2}{*}{ Sig } \\
Library environment & 40.4783 & 09.3443 & 322 & $.342^{* *}$ & .000 & \\
\hline \multicolumn{7}{|c|}{ * Sig. at .01 level }
\end{tabular}

It is shown in Table 6 that there was a significant relationship between the library environment and use of library $(r=.342, \mathrm{~N}=322, \mathrm{P}<$ .01). Hence, library environment had influenced the use of library by the students. The hypothesis is rejected.

Ho 2: There is no significant relationship between user education and use of library by undergraduates at the University of Ibadan

Data in Table 7 represents the correlation analysis showing the relationship between user education and use of library.

Table 7: Relationship between user education and use of library

\begin{tabular}{|l|l|l|l|l|l|l|}
\hline Variable & Mean & Std. Dev. & $\mathbf{N}$ & $\mathbf{R}$ & $\mathbf{P}$ & Remark \\
\hline Use of library & 32.9068 & 13.2833 & & & & \multirow{2}{*}{ Sig } \\
User education & 3.9814 & 2.1702 & 322 & $.191^{* *}$ & .001 & \\
\hline
\end{tabular}

It is shown in Table 7 that there was a significant relationship between user education and use of library $(r=.191, \mathrm{~N}=322, \mathrm{P}<.01)$. Hence, user education had influence on the use of library by these undergraduates. The hypothesis is rejected.

Ho 3: There is no joint effect of independent variables (library environment and user education) on the use of library by undergraduates of the University of Ibadan

Data in Table 8 represents the multiple regression analysis showing the joint contribution of library environment and user education on the use of library by the undergraduates. 
Table 8: Joint contribution of library environment and user education on the use of library

\begin{tabular}{|l|l|l|l|l|l|l|}
\hline R & \multicolumn{2}{|l|}{ R Square } & $\begin{array}{l}\text { Adjusted } \\
\text { R Square }\end{array}$ & $\begin{array}{l}\text { Std. Error of the } \\
\text { Estimate }\end{array}$ \\
\hline .362 & .131 & .125 & 12.4225 \\
\hline A N O V A \\
\hline Model & $\begin{array}{l}\text { Sum of } \\
\text { Squares }\end{array}$ & DF & $\begin{array}{l}\text { Mean } \\
\text { Square }\end{array}$ & F & Sig. & Remark \\
\hline $\begin{array}{l}\text { Regression } \\
\begin{array}{l}\text { Residual } \\
\text { Total }\end{array}\end{array} \begin{array}{l}7411.418 \\
49227.787\end{array}$ & $\begin{array}{l}2 \\
319\end{array}$ & $\begin{array}{l}3705.709 \\
154.319\end{array}$ & 24.013 & .000 & Sig. \\
\hline
\end{tabular}

Table 8 shows the joint contribution of the two independent variables (library environment and user education) to the prediction of the dependent variable (use of library). The Table shows a coefficient of multiple correlation $\left(\mathrm{R}=.362\right.$ and a multiple $\mathrm{R}^{2}$ of .131 . This means that $13.1 \%$ of the variance is accounted for by the two predictor variables when taken together. The significance of the composite contribution was tested at $\mathrm{P}<.05$. The Table also shows that the analysis of variance (ANOVA) for the regression yielded F-ratio of 24.013 (significant at 0.05 level). This implies that the joint contribution of the independent variables to the dependent variable was significant and that other variables not included in this model may have accounted for the remaining variance.

Ho4: There is no relative effect of library environment and user education on the use of library by undergraduates of the University of Ibadan

Multiple regression analysis was used to test the relative contribution of library environment and user education on the use of library by the undergraduates.

Table 9: Relative contribution of library environment and user education on the use of

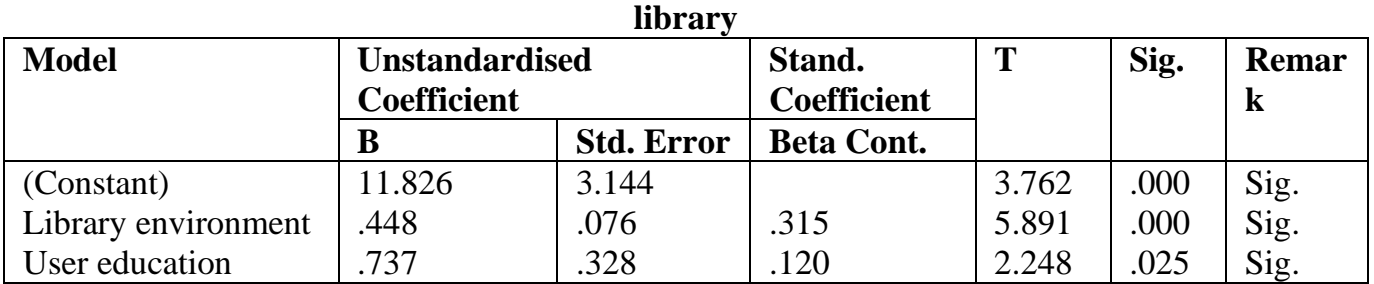

The relative contribution of the two independent variables to the dependent variable was expressed as beta weights: library environment $(\beta=$ $.315, \mathrm{P}<.05)$ and user education $(\beta=.120, \mathrm{P}<.05)$. It is noted from the results that library environment and user education independently and significantly predict the use of library among the undergraduates of University of Ibadan. 


\section{Discussion}

This section discusses the findings as revealed in the previous section based on the literature. The findings are presented according to the objectives of the study.

\section{Characteristics of library environment}

The study found that the characteristics of library environment in the University of Ibadan included good library building, seating arrangement, and availability of library information resources. Furthermore, library furniture, library personnel, temperature within the library, lighting, décor of the library (interior designs), signage within and outside the library, as well as noise level within the library were all rated as good by a sizeable numberof the respondents. This finding is a bit contrary to the findings of Amusa and Iyoro (2013) where most environmental indicators such as library building, availability and arrangement of furniture, temperature and décor of the libraries were rated as fair.

\section{Methods of delivering user education}

It was found that majority of the students were aware of user education in University of Ibadan library. This was supported by Amusa and Iyoro (2013) where 100\% respondents were aware of library instruction prgrammes in their institutions. The study also showed that library orientation for new students, use of library course (GES 101: Use of English), and guided library tour were the most popular user education programmes in the main library as indicated by the students. However, others like lectures, one-on-one library use instruction, and basic bibliographic instruction were not popular methods of user education delivered to undergraduates in the University. These findings were supported by Ilo and Idiegbeyan-ose (2011) who studied the various types of usereducation offered in Covenant University, which include library orientation, teaching of use of library and study skills, basic bibliographic instructions, etc. Suleiman (2012) also affirmed that library orientation and instruction were among the programmes used to enhance library skills to new students.

Obviously, research has shown that library orientation, guided tour and use of library course were the most popular methods of user education programmes in academic libraries. This was further buttressed by Maduako (2013) who found that the user education programmes of two colleges of education were structured in the form of library orientation, library tour, bibliographic instruction and user awareness. Amusa and Iyoro (2013) also found that most users were familiar with orientation talk and library course as the two main types of library instruction programmes in universities in Southwest Nigeria. 


\section{Purpose of library use among undergraduates}

The undergraduates in University of Ibadan used the library to study and read for examinations, find materials for assignments, and also to do research work, e.g. project and term paper. They also used the library to consult reference materials like encyclopedia, dictionaries; borrow materials for academic work; consult librarians for information; make use of the wireless internet connectivity (Wi-Fi) on their devices; access electronic information resources; make use of computers and internet access; as well as to consult newspapers. Meanwhile, only few of the students used the library for group discussion with course mates and for relaxation or sleeping respectively. The findings were somehow corroborated by Amusa and Iyoro (2013) which found that majority of the students (60\%) used the library to spend time between classes, to meet groups or socialize, to study or read their lecture notes, and also for research.

\section{Frequency of use of library among undergraduates}

The study revealed that the undergraduates mostly used the library daily or at least twice in a week to study and read for examination, as well as finding materials for assignments. It was further revealed that majority of the undergraduates never used the library for group discussion with course mates or to consult newspapers. It is observed that some undergraduates visit and use the library for as many times as possible in a day depending on certain motivating factors, some of which included availability of regular power supply to charge and use their devices (laptops, tabs/pads, smart phones, reading lamps, etc.) as well as stable internet access and relevant electronic resources.

\section{Challenges to the use of library}

Inadequate technological facilities to use electronic sources, poor network/internet connectivity to access electronic databases, and mostly outdated and non-current library collections were some of the major challenges of using the University library by the students. It was further shown that difficulty in locating materials from the shelves, poor lighting due to epileptic power supply, and low awareness of the information sources and services were also prominent among the challenges militating against the effective use of the library. This finding was supported by Edem, Ani and Ocheibi (2009) who submitted that undergraduate students experienced difficulty in locating items from the library collection and did not understand the processes for retrieving journal articles.

Lamptey (2010) further affirmed that lack of modern techniques of teaching and crowdedness and largeness of the class were problems affecting user education and use of library resources and services among 
undergraduates in academic libraries. The findings also coincided with Suleiman's (2012) assertion that university students are seriously facing problems that stresses independence of thoughts and actions. They don't know how to deal with new environment; they need careful and special guides which help them to deal with various sources of information independently.

\section{Relationships between library environment, user education and use of library}

The study found a significant relationship between the library environment and use of library among undergraduates in University of Ibadan. This means that library environment had influenced the use of library by the students. According to Juceviciene and Tautkeviciene (2004), students' use of the library is significantly influenced by their predispositions regarding the library as a learning environment as well as by the study educational environment. Likewise, there was a significant relationship between user education and use of library among undergraduates in University of Ibadan. Maduako (2013) reported that a moderate positive relationship exists between user education and library use. Suleiman (2012) reported that about $96 \%$ of university undergraduates believe that user education programme helped them to make effective use of the OPAC and other library resources and services.

The present study also revealed that both library environment and user education jointly contributed to the dependent variable (use of library). Likewise, library environment and user education independently and significantly predicted the use of library among the undergraduates. These findings were strongly supported by Amusa and Iyoro (2013) who found that about $76 \%$ of their respondents affirmed that the library environment had a great influence on library use. Suleiman (2012) further corroborated these findings that user education programmes in the library helps the users to find relevant information through Online Public Access Catalogue and other information sources in the library.

\section{Conclusion}

The study established that undergraduates' use of the library largely depend on the various characteristics that make up the University library. User education is very important in academic library services because it determines the extent to which students would patronize and use the library resources and services. User education programmes such as orientation, use of library courses and guided tour enable the library to sensitise the students and create adequate awareness about the available resources and services of the library to meet and satisfy the needs of the users. 
The library environment and user education, either jointly or independently, are great determinants of library use among any group of users in a library. For instance, while a library with adequate seating space, adequate and up-to-date information resources, good customer services, stable electricity supply and internet access (both within and outside the library) will always attract users to the library, users will feel so reluctant to patronize a library with poor internal and external environment. Likewise, user education programme that is well organized will create confidence in the users, and therefore, encourage them to use the library effectively.

\section{Recommendations} the study:

The following recommendations were made based on the findings of

1. Considering the importance of user education to the use of library, it is recommended that user education should be given more priority in the University library in order to sensitise more students and draw their attention to the available resources and services that can be of immense benefit to them in the course of their studies.

2. Students would not use the library due to the poor nature of most resources and facilities within and outside the library. Therefore, the environment of academic libraries (both internal and external) should be made more conducive such that can always attract the users to the library. Beautification of library environment and investments in new technologies by the library management can add glamour to the library environment, and, hence attract users to the library always.

3. Library information resources and services are created for the use of students, so students should form a habit to visit the library always in order to access the collections and services which are specifically provided to support their learning and research activities.

4. Students should always create time to attend user education programmes organized by the university libraries, either as orientation or special training sessions on new services. This will afford them the opportunity to acquaint themselves with the general library environment which will further encourage effective library use.

\section{References}

Abubakar, A. (2003). College of Education libraries: the need for more emphasis on user education. Isa Kaita. Multidisciplinary Journal of Education 1.1: 15-20 
Abubakar, U and Isyaku, A. (2012). Teaching Information Literacy Skills in Nigerian Universities: Whose Responsibility? Journal of Research in Education and Society;3

Adeniran, P. (2011). User satisfaction with academic libraries services: Academic staff and students perspectives. International Journal of Library and Information Science, 3.10: 209-216.

Adeoye, A. O. and Elegunde, A. F. (2012). Impacts of External Business Environment on Organisational Performance in the Food and Beverage Industry in Nigeria. British Journal of Arts and Social Sciences 6.2: 194201. Retrieved from http://www.bjournal.co.uk/BJASS.aspx

Aina, L.O. (2002). Research in Information Science: An African Perspective (ed.). Stirling Horden Publishers (Nig.) Ltd

Akinbola, O. O. (2007). Significance of User Education Programme on the Use of library. International Journal of Research in Education 4.1/2: 188193.

Amusa, O.I and Iyoro, A.O. (2013). Influence of Library Environments, Instructional Programmes, and User-Librarian Collaborations on Library Use by Undergraduate Students in Nigeria. Chinese Librarianship: an International Electronic Journal 35: 72-86 URL: www.iclc.us/cliej/c135AI.pdf

Association of College and Research Libraries (ACRL). (2011). Standards for distance learning libraries services. Retrieved on July 14 from http://www.ala.org/ala/mgrps/divs/acrl/standards/guidelinesdistancelearning. cfm

Chowdhury, G. (2006). Qualitative Research for the Information Professional: A Practical Handbook $\left(2^{\text {nd }}\right.$ ed.). Online Information Review. Vol 30 Issue 4

Edem, N., Ani, O. and Ocheibi, J. A. (2009). Students' perceived effectiveness in the use of library resources in Nigerian universities. Educational Research and Review 4.6: 322- 326. Available online at http://www.academicjournals.org/ER

Emwanta and Nwalo, K.I.N. (2013). Influence of computer literacy and subject background on use of electronic resources by undergraduate students in universities in South-western Nigeria. International Journal of Library and Information Science 5.2: 29-42, Available online at http://www.academicjournal.org/IJLIS

Ilo, P.L and Idiegbeyan-ose, J. (2011). Library User Education Programmes for Covenant University Freshmen: Impact, Challenges and Possible Solutions. Samaru Journal of Information Studies. 11.1 \& 2: 7-12 Jucevicine, P and Tautkeviciene, G. (2004). The library learning environment as a part f university educational environment. Paper presented at the European Conference on Educational Research, University of Crete, 
22-25 September 2004. Retired $5^{\text {th }}$ May, 2015 from: http://www.leeds.ac.uk/educol/documents/00003737.htm

Krejcie, R.V. and Morgan, D.W. (1970). Determining sample size for research activities. Educational and Psychological Measurement 30: 607610.

Lamptey, R.B. (2010). Promoting effective use of the Library resources and services at Kwame Nkrumah University of Science and Technology Library, Kumasi, Ghana. Ghana Library Journal. 42. 1 \& 2.

Maduako, P.U. (2013). User education and library use in college of education in Abia and Imo States. Library Philosophy and Practice (ejournal). Paper 955. Retrieved January 8, 2015 from http://digitalcommons.unl.edu/libphilprac/955

Njanja, W.L. (2009). An investigation into management Strategies affecting performance of Micro, Small and medium Enterprises in Kenya. Unpublished thesis. University of South Africa.

Ojedokun, A.A. (2000). Library support services for distance education in Southern Africa. Retrieved on April 19, 2014, from http://www.unisanet.unisa.edu.au/ccc/papers/refereed/paper30/paper301.htm

Suileiman, S.A. (2012). User Education Programmes in Academic Libraries: The Experience of the International Islamic University Malaysia Students. Retrieved on April 22, 2014, from www.webpages.uidaho.edu mbolin/ali.htm

University of Ibadan Pocket Statistics. (2011). Academic Planning Unit, University of Ibadan.

Unwin, L., Bolton, N., Stephens, K. (1997). The Use of Libraries by Postgraduate Distance Learning Students: a mismatch of expectations. The Journal of Open, Distance and E- learning. Vol. 12 Issue 3 Wagner, M. (2005). Sustainability and competitive advantage: empirical evidence on the influence of strategic choices between environmental management approaches. Environmental Quality Management, 14.3: 3148 